\title{
Performance of Data Mining Classifiers on Kolb's Learning Style Inventory (KLSI)
}

\author{
P. Menaka and K. Nandhini \\ Department of PG and Research in Computer Science, Dr. N.G.P. Arts and Science College Chikanna \\ Government Arts College, Konganagiri, Vivekananda Nagar - 641602, Tirupur, Tamil Nadu, \\ India; menakasdream@gmail.com, krishnandhini@yahoo.com
}

\begin{abstract}
Objectives: This study is intended to examine the effectiveness of predicting the learning style of college students. To understand experiential learning, many have reiterated the need to be able to identify students' learning styles. Kolb's Learning Style Model is the most widely accepted learning style model and has received a substantial amount of empirical support. Kolb's Learning Style Inventory (LSI), although one of the most widely utilized instruments to measure individual learning styles, possesses serious weaknesses. Methods/Statistical Analysis: The proposed work introduces the study of efficiency in Kolb's learning style. Classification algorithms used in this research include J48, BayesNet, and Naïve Bayes and Random forest classifier. The data was collected from 30 students in Department of Information Technology, Dr. N.G.P. Arts and Science College in the final semester of academic year 2018. The 10-fold Cross Validation was used to create and test the model and the data was analysed by the WEKA program. Questionnaires were distributed to participants in class and then collected by the researcher after participants had finished them. Findings: After all the questionnaires were collected, data was then input in the computer and then statistically analysed. The J48, BayesNet, Naïve Bayes and Random forest classifiers are used to measure the classification accuracy. In order to measure the learner style results the metrics like precision, recall, accuracy and Kappa values are used in this work. Application/Improvements: The result shows that random forest algorithm has more capability in predicting learning style of the students. Understanding the learning style of students is the first step and it may give a fruitful result when it is embedded with learning environment.
\end{abstract}

Keywords: Kolb’s Learning Style Inventory (LSI), J48, NB Tree and Naïve Bayes

\section{Introduction}

The cognitive psychology based measure of a person through processing of information, mode of active learning and pattern of information are referred to as learning styles. It means that a person may prefer specific learning style while learning a subject. Sometimes the learners may use mixed learning style. The progression of information technology such as internet surged the growth of online educational programs which change the traditional system of education. The emergence of technology has become a competitive advantage for higher education institutions as it can provide an alternative approach in providing better quality of learning. Many institutions are responding to the pressures by embracing the new technologies. Technology has a vital role to play in building up 21st century skills, broadening access to education and personalizing the learning experience to adapt teaching to the unique needs of each learner.

One important aspect of learning style is to recognize the learning style of the student and measure the impact on their academic achievement. According to the knowledge of learning styles can be used to increase students' self-awareness and meta-cognition of their strengths and weaknesses. In argues that most of the students are unaware of their learning style and they are mostly unlikely to start learning in new ways. 
Traditionally, to classify individual differences, an implemented learning style to analyze a learner's individual competence was applied. The learning styles are the perception and the learning each learner individually possesses, which are integrated with a learner's physical appearance, emotion, and perception and these show how effective each learner learns. Moreover, the learning styles are considered changeable habits. Nowadays, there exists a learner-centered education system in Tamil Nadu. The instructors have to base the contents and activities on interests and skills of individual learners. Accordingly, the learning style of each learner has an important effect on learning performance of the learners. In this research, the theory of David Kolb's learning style was applied.

\section{Literature Survey}

$\mathrm{In}^{1}$ studied about the adaptability of learning style in the general educational practice. The instructional method plays an important role while assessing the learning style. $\mathrm{I} n=2$ examined the performance of learning styles into an adaptive e-learning hypermedia. The result depicts that the learning style based coaching is significantly influences on the academic achievement of students. $\mathrm{In}^{3}$ studied the dynamic learning style prediction based on the pattern recognition technique. This system works as middleware for other intelligent tutoring system and it can process topic-dependent data to make prediction, update learning style profiles in a recursive manner.

In ${ }^{4-6}$ identified the learning style in Learning Management System (LMS). In ${ }^{7}$ detecting the learning style in LMS using different data mining techniques. The J48 classifier is out performed in the experimental results. $\mathrm{In}^{8}$ examined the recognition of learning style using artificial neural network in an e-learning platform. It uses adaptive characteristics in the e-learning environment, and it helps to predict the learning style more accurately. In $-\underline{-12}$ examined the students' attitudes and their learning styles using KLSI in mobile learning context. The result reveals that major part of the students are classified under Converger learning style and also found highest attitude towards mobile learning.

In $\underline{13,14}$ examined in-depth analysis of FelderSilverman based learning style. The analysis result shows the most representative learning styles, which is also can incorporate in the technology enhanced learning environments. In ${ }^{15}-\underline{18}$ examined the adoption of student learning styles in the web-based educational environment and it uses Felder-Silverman Learning Style Theory.

In $\frac{19}{}$ compared the e-learning environment with traditional classroom. Both environments enable has its own way of assessing the learning style. It means significant differences found between two models. $\operatorname{In}^{20}$ considered an analysis of the delivery methods using traditional learning, eLearning, and blended learning. Analysis of the comparison between traditional learning and eLearning has been done. The characteristics and benefits of blended learning are analyzed. Blended learning course development steps are described.

$\mathrm{In}^{21-23}$ proposed a learning style classification mechanism to classify and then identify students' learning styles. The proposed mechanism improves $\mathrm{k}$-nearest neighbor (k-NN) classification and combines it with Genetic Algorithms (GA). The experimental results indicate that the proposed classification mechanism can effectively classify and identify students' learning styles. $\mathrm{In}^{24}$ evaluated the possibility of automatic detection of learning style in the e-learning platform. The NB Tree classification algorithm is used with Binary Relevance classifier. The result states that the automatic learning style is predictable and consistent. In ${ }^{25}$ incorporates the Self Organizing Map (SOM) based neural networks for recognizing the learning styles under different pedagogical models. It is a part of Intelligent Tutoring Systems compatible for mobile and web-based environment. $\mathrm{In}^{26-27}$ examined the adaptive e-learning model, which is suitable for predicting the learning style of the students. Bayes, Decision Tree and SVM classifiers are used to measure the accuracy of learning style prediction. Further, the result states that J48 classifier produces better results.

\section{Learning Style Measures}

The classification of large data sets is an essential problem in machine learning. For a database with millions of records and more number of classes and each record belongs to one of the given classes. In this context, the classification problem is to determine the appropriate class for the record. In a supervised type of classification, a set of records called training data which means the strong dependency of the class. In the training set, the classification process attempts to generate the descriptions of the classes, and these descriptions help to classify the unknown records. There are several approaches to 
supervised classifications. In this study, J48, BayesNet, Naïve Bayes and Random forest classifiers are used to examine the classification accuracy of Kolb' learning style.

\subsection{Kolb's Learning Style Model}

Seven learning style models exist, and David Kolb's model is more famous among the list. Moreover, the Kolb Learning Style Inventory (KLSI) method is more suitable for our focused group and still active in the current scenario. This paper uses KLSI version 3.0 based assessment method which has four categories such as Divergent, Assimilator, Converger, and Accommodator.

The Divergent learning style is suitable for individuals possessing concrete experience and reflective observation. This type of individuals is capable of assessing an event from different angles. They are more like observers rather than activists.

The Assimilator learning style includes abstract conceptualisation and reflective observation. Individuals bearing the characteristics of this type of learning are capable of grasping a large scale of information scattered over a broad discipline and convert it to a logical whole. Instead of dealing with other individuals, they prefer to deal with abstract concepts and issues. They generally focus on the logical validity of theories instead of their applicability.

The Converger learning style describes an individual prefers both abstract conceptualisation and active experimentation. Individual learning in this way is quite successful regarding the practical application of ideas and theories, solving problems and making decisions. They prefer technical tasks and problems to social relations.

The Accommodator learning style includes concrete experience and active experimentation. An individual who prefer this learning style will learn by doing and feeling. They like new experiences and planned work. Instead of intellectual analysis, they prefer to act on feelings. They like collecting information through dialogues with people, rather than gathering the necessary information through technical analysis. To validate the accuracy of predicting the learning style four data mining classifier algorithms were used such as BayesNet, J48, and Naive Bayes and Random forest classifier.

\subsection{J48 Decision Tree Classifier}

Classification is a process of model construction, which assigns class labels to the respective records according to the closest match specification. The J48 is a decision tree algorithm, which is used to create univariate decision trees. Among the list of independent and dependent variables in the dataset, the $\mathrm{J} 48$ algorithm helps to predict the target variable for the new record. This algorithm generates the rules for the prediction of the target variable. With the help of the tree classification algorithm, the critical distribution of the data is easily understandable ${ }^{28}$.

The J48 algorithm is an extension of the ID3 algorithm, which contains the additional features such as missing values, continuous attribute value ranges, decision tree pruning, and derivation of rules. The pruning logic is precisely used to manage the over fitting problem. This algorithm generates rules from the specific identity of the data. The main aim is the generalisation of a decision tree until it gains equilibrium of flexibility and accuracy.According to ${ }^{29}$ steps of the algorithm defined as follows: 1 . if the instances belong to the same class, the tree considered as a leaf and returned the label as same class, 2. the potential information is computed for each attribute and returns the gain obtained from the result, and 3. finally, the best attribute is estimated using the present selection criterion and the attribute selected for branching.

\subsection{Random Forest Classifier}

The random forest classifier contains a group of tree classifiers, where each classifier is generated using a random vector sampled independently from the input vector. Further, each tree forms a group of choice for the favourite class to classify the input vector ${ }^{30}$. The random forest classifier used in this study consists of using randomly selected features or a combination of features at each node to grow a tree. Bagging method is used to generate the training dataset randomly from the specific number of the original training set. The design of a decision tree is mainly based on the attribute selection measure and the pruning method.

There are many approaches to the selection of attributes used for decision tree induction and most approaches assign a quality measure directly to the attribute. The most frequently used attribute selection measures in decision tree induction are the Information Gain Ratio criterion 31 and the Gini Index. The random forest classifier uses the Gini Index as an attribute selection measure, which estimates the impurity of an attribute reference to the specific classes. For a given training set $\mathrm{T}$, selecting one 
case (pixel) at random and saying that it belongs to some class $\mathrm{Ci}$, the Gini index can be written as:

$$
\sum \sum_{j \neq i}\left(\frac{f\left(C_{i}, T\right)}{|T|}\right)\left(\frac{f\left(C_{j}, T\right)}{|T|}\right)
$$

Where $\mathrm{f}(\mathrm{Ci}, \mathrm{T}) /|\mathrm{T}|$ is the probability that the selected case belongs to class $\mathrm{Ci}$. Each time a tree is grown to the maximum depth on new training data using a combination of features. These fully grown trees are not pruned, which is one of the significant advantages of the random forest classifier compared to the decision tree method. The number of features used at each node to generate a tree and the number of trees to be grown is two user-defined parameters required to generate a random forest classifier. At each node, only selected features are searched for the best split. Thus, the random forest classifier consists of $\mathrm{N}$ trees, where $\mathrm{N}$ is the number of trees to be grown, which can be any value defined by the user. For a new dataset, each case of the datasets is passed down to each of the $\mathrm{N}$ trees. The forest chooses a class having the most out of $\mathrm{N}$ votes, for that case.

\subsection{BayesNet Classifier}

BayesNet is a Bayesian Network derives from Bayes Theorem. It is also known as probabilistic directed acyclic graphical model, which represents a set of variables and its dependencies. It contains a set of observable quantities, latent variables and unknown parameters. The edges in the graph represent the conditional dependencies and the nodes which are not connected are referred to as conditionally independent variables. The one greatest advantage of using Bayesian networks is that it is easy to understand by the human. Further, it can save considerable amounts of memory.

\subsection{Naive-Bayes Classifier}

In classification learning, the Naive Bayes classifier is a straightforward method, which is described by a vector of attribute values. It is also referred to as a probabilistic classifier and contains training, testing phases. The training data has a set of instances to train the classifier. Similarly, the testing data also has a group of instances, which validates the given instance with training data to conclude. This classifier defined as follows;

- $\mathrm{C}$ is a random variable, which denotes the class of an instance,
- $\mathrm{X}<\mathrm{X}_{1}, \mathrm{X}_{2}, \ldots . ., \mathrm{X}_{\mathrm{k}}>$ is a vector, which representing observed attribute values,

- $\mathrm{c}$ as a specific class,

- $\mathrm{x}<\mathrm{x}_{1}, \mathrm{x}_{2}, \ldots . . \mathrm{x}_{\mathrm{k}}>$ as a specific observed attribute value vector, and

- $\mathrm{X}=\mathrm{x}$ as shorthand for $\mathrm{X}_{1}=\mathrm{x}_{1} \mathrm{X}_{2}=\mathrm{x}_{2} \wedge \cdots \wedge \mathrm{X}_{\mathrm{k}}=\mathrm{x}_{\mathrm{k}}$.

The classification error can be minimized through the selection of $\operatorname{argmax}_{c}(\mathrm{p}(\mathrm{C}=\mathrm{c} \mid \mathrm{X}=\mathrm{x}))$ for each $\mathrm{x}$. The Bayes' theorem is used to compute the probability:

$$
p(C=c \mid X=x)=\frac{p(C=c) p(X=x \mid C=c)}{p(X=x)}
$$

Since the denominator in Eq. (1) is invariant across classes, it does not affect the final choice and can be dropped, thus:

$$
p(C=c \mid X=x) \propto p(C=c) p(X=x \mid C=c)
$$

and need to be examined from the training data. In this context, $\mathrm{x}$ is an unseen instance, which does not appear in the training data and it may not be possible to directly estimate. Hence, a simplification is made: if attributes $\mathrm{X}_{1}, \mathrm{X}_{2}, \ldots, \mathrm{X}_{\mathrm{k}}$ are conditionally independent of each other given the class, then

$$
\begin{gathered}
p(X=x \mid C=c)=p\left(\wedge X_{i}=x_{i} \mid C=c\right) \\
=\prod p\left(X_{i}=x_{i} \mid C=c\right)
\end{gathered}
$$

Combining Eq. (2) and (3), one can further estimate the probability by:

$$
p(C=c \mid X=x) \propto p(C=c) \prod p\left(X_{i}=x_{i} \mid C=c\right)
$$

Classifiers using Eq. (5) are called naive-Bayes classifiers. Naive-Bayes classifiers are simple, efficient and robust to noisy data. One limitation is that the attribute independence assumption in Eq. (4) is often violated in the real world.

\section{Results and Discussion}

The Kolb's learning style prediction is implemented in PHP, which is used as a source for collecting the data. The data employed in this study was collected from 30 students in the Department of Computer Science, Dr. N.G.P. Arts and Science College during the academic year 2018. Four classifiers such as BayesNet, J48, Naive Bayes and Random forest are used to measure the efficiency in predicting the learning style classification. The subsequent depicts the 
Precision, Recall and Accuracy of the selected classifiers. In this work, 30 instances are taken as input with 11 attributes, in which the first attribute denotes name of the student and last attribute denotes the class label and the remaining attributes are learning style questionnaire.

The depicts precision result of selected classifiers on the Kolb's learning style inventory of the students. The average precision of each classifier is taken into account, which shows the result as follows; $\mathrm{BN}$ value is $69.91 \%$, J48 value is $68.90 \%$, NB value is $70.09 \%$ and RF value is $72.32 \%$.

The Figure 1 depicts the graphical representation of the precision metrics obtained from the selected classifiers. It is observed from the result that Random Forest classifier has shown the highest precision compared to other classifiers considered in this study.

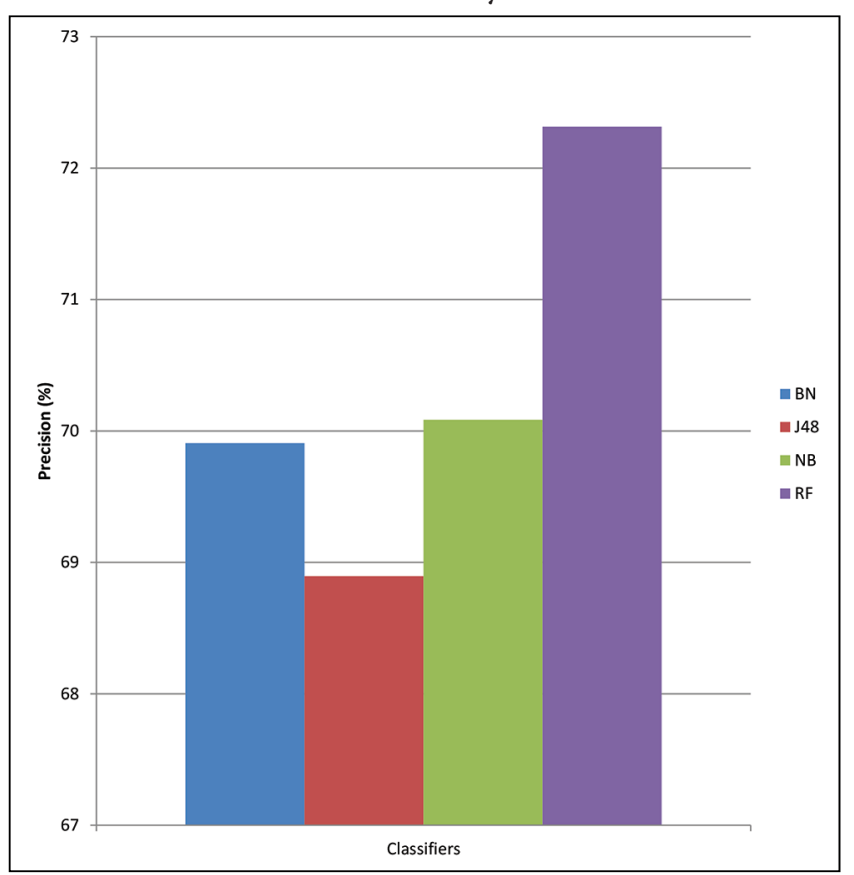

Figure 1. Precision metrics with four classifiers.

The depicts recall result of selected classifiers on the Kolb's learning style inventory of the students. The average recall of each classifier is taken into account, which shows the result as follows; $\mathrm{BN}$ value is $63.84 \%$, J48 value is $66.96 \%$, NB value is $72.76 \%$ and RF value is $72.32 \%$.

The Figure 2 illustrates the graphical representation of the recall metrics obtained from the selected classifiers. It is observed from the result that Naïve Bayes classifier is outperforming in the recall perspective.

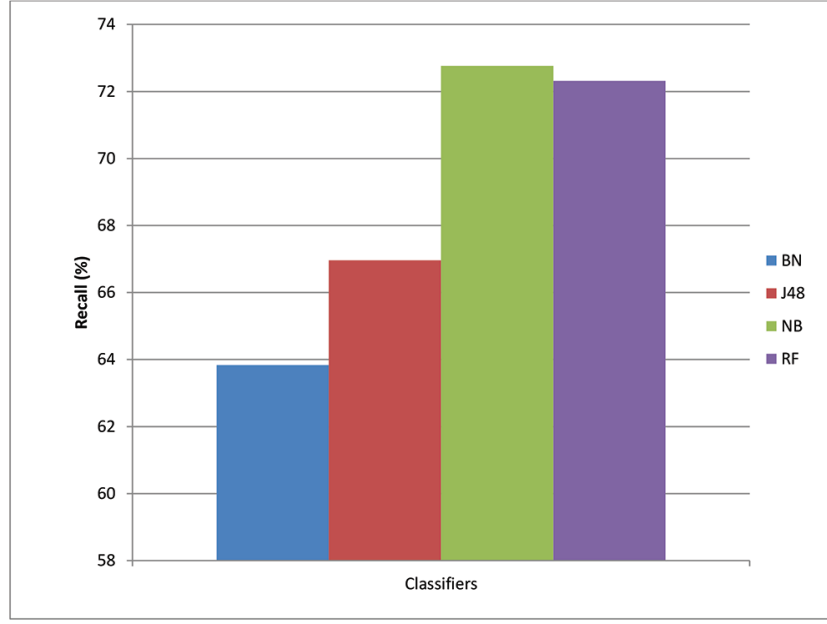

Figure 2. Recall metrics with four classifiers.

The exhibits the classification accuracy of selected classifiers reference to Kolb's learning style inventory of the students. It is observed from the result that the average accuracy of $\mathrm{BN}$ is $66.66 \%$, J48 is $70 \%, \mathrm{NB}$ is $73.33 \%$ and $\mathrm{RF}$ is $76.66 \%$. It means that the random forest classifier is producing more accuracy compared to other classifiers considered in this study.

The Figure 3 illustrates the graphical representation of an average accuracy obtained from the selected classifiers.

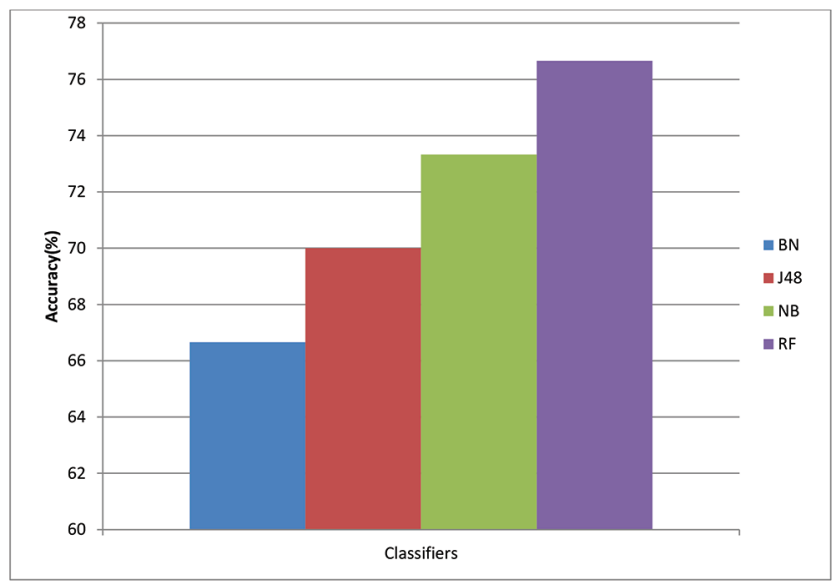

Figure 3. Accuracy metrics with four classifiers.

The Figure 4 illustrates the kappa analysis of Kolb's learning style inventory based classification of students. The kappa value indicates inter-rater agreement of each classifier and the result shows that random forest has more agreement in predicting the learning style. 


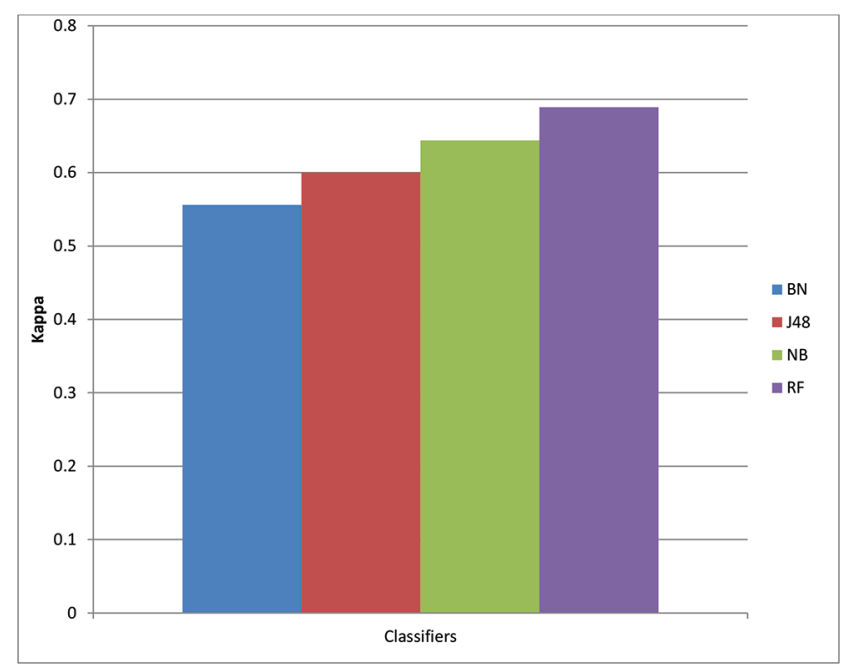

Figure 4. Kappa value with four classifiers.

\section{Summary and Conclusion}

The learning style plays an important role in classifying the students. Among the list of learning style models available, David Kolb's learning style inventory is quite famous and suitable for this study. The primary questionnaire was designed and implemented as PHP application and given access to students for data collection. This study is intended to examine the effectiveness of predicting the learning style of college students. The J48, BayesNet, Naïve Bayes and Random forest classifiers are used to measure the classification accuracy. The result shows that random forest algorithm has more capability in predicting learning style of the students. Understanding the learning style of students is the first step and it may give a fruitful result when it is embedded with learning environment. Thus, future research may incorporate the automatic learning style prediction over specific learning environment.

\section{References}

1. Pashler H, McDaniel M, Rohrer D, Bjork R. Learning styles: Concepts and evidence, Psychological Science in the Public Interest. 2008; 9(3):105-19. https:// doi.org/10.1111/j.1539-6053.2009.01038.x. $\quad$ PMid: 26162104.

2. Mustafa YEA, Sharif SM. An approach to Adaptive E-Learning Hypermedia System based on Learning Styles (AEHS-LS): Implementation and evaluation, International Journal of Library and Information Science. 2011; 3(1):15-28.
3. Yang J, Huang ZX, Gao YX, Liu HT. Dynamic learning style prediction method based on a pattern recognition technique, IEEE Transactions on Learning Technologies. 2014; 7(2):165-77. https://doi.org/10.1109/TLT.2014.2307858.

4. Enabling learning management systems to identify learning styles. Date accessed: 2006. https://www.researchgate. net/publication/239552304_Enabling_Learning_ Management_Systems_to_Identify_Learning_Styles.

5. García P, Amandi A, Schiaffin, S, Campo M. Evaluating Bayesian networks' precision for detecting students' learning styles, Computers and Education. 2007; 49(3):794808. https://doi.org/10.1016/j.compedu.2005.11.017.

6. Truong HM. Integrating learning styles and adaptive e-learning system: Current developments, problems and opportunities, Computers in Human Behaviour. 2016; 55:1185-93. https://doi.org/10.1016/j.chb.2015.02.014.

7. LiyanagePP,GunawardenaL,HirakawaM.Detectinglearning styles in learning management systems using data mining, Journal of Information Processing. 2016; 24(4):740-49. https://doi.org/10.2197/ipsjjip.24.740.

8. Kolekar SV, Sanjeevi SG, Bormane DS. Learning style recognition using artificial neural network for adaptive user interface in e-learning. IEEE International Conference on Computational Intelligence and Computing Research; 2010. p. 1-5. https://doi.org/10.1109/ICCIC.2010.5705768. PMid: 19424966.

9. Investigation of higher education students' learning styles and attitudes towards mobile learning according to various variables. Date accessed: 07/2018. https://www. researchgate.net/publication/326557559_Investigation_ of_higher_education_students'_learning_styles_and_ attitudes_towards_mobile_learning_according_to_ various_variables.

10. Franzoni AL, Assar S, Defude B, Rojas J. Student learning styles adaptation method based on teaching strategies and electronic media. IEEE International Conference on Advanced Learning Technologies; 2008. p. 778-82. https:// doi.org/10.1109/ICALT.2008.149.

11. Kolb A, Kolb DA. Kolb's learning styles. In: Encyclopedia of the Sciences of Learning; 2012. p. 1698-703.https://doi. org/10.1007/978-1-4419-1428-6_228.

12. Adaptation to learning styles in e-learning: Approach evaluation. Date accessed: 01/2006. https://www. researchgate.net/publication/228799136_Adaptation_to_ learning_styles_in_e-learning_Approach_evaluation.

13. Graf S, Viola SR, Leo T, Kinshuk. In-depth analysis of the Felder-Silverman learning style dimensions, Journal of Research on Technology in Education. 2007; 40(1):79-93. https://doi.org/10.1080/15391523.2007.10782498.

14. Firte AA, Bratu CV, Cenan C. Intelligent component for adaptive E-learning systems. IEEE 5th International 
Conference on Intelligent Computer Communication and Processing, 2009. p. 35-38. https://doi.org/10.1109/ ICCP.2009.5284788.

15. Bajraktarevic N, Hall W, Fullick P. Incorporating learning styles in hypermedia environment: Empirical evaluation. In: Proceedings of the Workshop on Adaptive Hypermedia and Adaptive Web-Based Systems; 2003. p. 41-52.

16. Adaptation to student learning styles in web based educational systems. Date accessed: 2004. https://www. learntechlib.org/p/12978/.

17. Park H. Design and development of a mobile learning management system adaptive to learning style of students. IEEE International Workshop on Wireless and Mobile Technologies in Education (WMTE'05); 2005. p. 67-69.

18. Yang J, Liu H, Huang Z. Smap: To generate the personalized learning paths for different learning style learners. In: International Conference on Technologies for E-Learning and Digital Entertainment; 2010. p. 13-22. https://doi. org/10.1007/978-3-642-14533-9_2.

19. Babić S. E-learning environment compared to traditional classroom. In: MIPRO, 2011 Proceedings of the 34th International Convention; 2011. p. 1299-304.

20. Blended learning: The integration of traditional learning and eLearning. Date accessed: 10/2011. https://www. researchgate.net/publication/254014870_Blended_ learning_The_integration_of_traditional_learning_and_ eLearning.

21. Chang YC, Kao WY, Chu CP, Chiu CH. A learning style classification mechanism for e-learning, Computers and Education. 2009; 53(2):273-85. https://doi.org/10.1016/j. compedu.2009.02.008.

22. Dagez HE, Baba MS. Applying neural network technology in qualitative research for extracting learning style to improve e-learning environment. 2008 International Symposium on Information Technology; 2008 1. p. 1-6. https://doi.org/10.1109/ITSIM.2008.4631550.

23. DeCoux VM. Kolb's learning style inventory: A review of its applications in nursing research, Journal of
Nursing Education. 2016; 29(5):202-07. https://doi org/10.3928/01484834-19900501-04. PMid: 2162927.

24. Özpolat E, Akar GB. Automatic detection of learning styles for an e-learning system, Computers and Education. 2009; 53(2):355-67. https://doi.org/10.1016/j. compedu.2009.02.018.

25. Zatarain-Cabada R, Barrón-Estrada ML, Angulo VP, García AJ, García CAR. A framework for creating, training, and testing self-organizing maps for recognizing learning styles. In: International Conference on Technologies for E-Learning and Digital Entertainment; 2010. p. 53-64. https://doi.org/10.1007/978-3-642-14533-9_6.

26. A novel adaptive e-learning model matching educatorstudent learning styles based on machine learning. Date accessed: 11/10/2017. https:/www.researchgate.net/ publication/316753485_A_novel_adaptive_e-learning_ model_matching_educator-student_learning_styles_ based_on_machine_learning.

27. Akbulut Y, Cardak CS. Adaptive educational hypermedia accommodating learning styles: A content analysis of publications from 2000 to 2011, Computers and Education. 2012; 58(2):835-42. https://doi.org/10.1016/j. compedu.2011.10.008.

28. Nadali A, Kakhky EN, Nosratabadi HE. Evaluating the success level of data mining projects based on CRISP-DM methodology by a Fuzzy expert system. International Conference on Electronics Computer Technology; 2011 6. p. 161-65. https://doi.org/10.1109/ICECTECH.2011.5942073.

29. C4. 5 algorithm and multivariate decision trees. Date accessed: 2006. https://www.researchgate. net/publication/267945462_C45_algorithm_and_ Multivariate_Decision_Trees.

30. Random forests-random features. Date accessed: 09/1999. https://www.stat.berkeley.edu/ breiman/random-forests. pdf.

31. C4.5: Programs for Machine Learning. Date accessed: 1993. https://dl.acm.org/citation.cfm?id = 152181 . 